\title{
Association between Oxidative Stress and Outcome in Different Subtypes of Acute Ischemic Stroke
}

\author{
Nai-Wen Tsai, ${ }^{1}$ Ya-Ting Chang, ${ }^{1,2}$ Chi-Ren Huang, ${ }^{1}$ Yu-Jun Lin, ${ }^{2,3}$ \\ Wei-Che Lin, ${ }^{4}$ Ben-Chung Cheng, ${ }^{2,5}$ Chih-Min Su, ${ }^{2,6}$ Yi-Fang Chiang, ${ }^{1}$ Shu-Fang Chen, ${ }^{1,3}$ \\ Chih-Cheng Huang, ${ }^{1}$ Wen-Neng Chang, ${ }^{1}$ and Cheng-Hsien Lu ${ }^{1,2}$ \\ ${ }^{1}$ Department of Neurology, Chang Gung Memorial Hospital, Kaohsiung Medical Center, \\ Chang Gung University College of Medicine, 123, Ta Pei Road, Niao Sung District, Kaohsiung, Taiwan \\ ${ }^{2}$ Department of Biological Science, National Sun Yat-Sen University, Taiwan \\ ${ }^{3}$ Department of Neurosurgery, Chang Gung Memorial Hospital, Kaohsiung Medical Center, \\ Chang Gung University College of Medicine, 123, Ta Pei Road, Niao Sung District, Kaohsiung, Taiwan \\ ${ }^{4}$ Department of Radiology, Chang Gung Memorial Hospital, Kaohsiung Medical Center, \\ Chang Gung University College of Medicine, 123, Ta Pei Road, Niao Sung District, Kaohsiung, Taiwan \\ ${ }^{5}$ Department of Internal Medicine, Chang Gung Memorial Hospital, Kaohsiung Medical Center, \\ Chang Gung University College of Medicine, 123, Ta Pei Road, Niao Sung District, Kaohsiung, Taiwan \\ ${ }^{6}$ Department of Emergency Medicine, Chang Gung Memorial Hospital, Kaohsiung Medical Center, \\ Chang Gung University College of Medicine, 123, Ta Pei Road, Niao Sung District, Kaohsiung, Taiwan \\ Correspondence should be addressed to Nai-Wen Tsai; tsainw@yahoo.com.tw
}

Received 27 February 2014; Accepted 7 April 2014; Published 8 May 2014

Academic Editor: Hung-Chen Wang

Copyright (C) 2014 Nai-Wen Tsai et al. This is an open access article distributed under the Creative Commons Attribution License, which permits unrestricted use, distribution, and reproduction in any medium, provided the original work is properly cited.

Objectives. This study investigated serum thiobarbituric acid-reactive substances (TBARS) and free thiol levels in different subtypes of acute ischemic stroke (AIS) and evaluated their association with clinical outcomes. Methods. This prospective study evaluated 100 AIS patients, including 75 with small-vessel and 25 with large-vessel diseases. Serum oxidative stress (TBARS) and antioxidant (thiol) were determined within 48 hours and days 7 and 30 after stroke. For comparison, 80 age- and sex-matched participants were evaluated as controls. Results. Serum TBARS was significantly higher and free thiol was lower in stroke patients than in the controls on days 1 and 7 after AIS. The level of free thiol was significantly lower in the large-vessel disease than in the small-vessel disease on day 7 after stroke. Using the stepwise logistic regression model for potential variables, only stroke subtype, NIHSS score, and serum TBARS level were independently associated with three-month outcome. Higher TBARS and lower thiol levels in the acute phase of stroke were associated with poor outcome. Conclusions. Patients with large-vessel disease have higher oxidative stress but lower antioxidant defense compared to those with small-vessel disease after AIS. Serum TBARS level at the acute phase of stroke is a potential predictor for three-month outcome.

\section{Introduction}

Stroke is a major cause of morbidity and mortality worldwide [1]. Inflammation and oxidative stress play important roles in acute ischemic stroke (AIS) [2-4] and the close relationship between inflammation and oxidative stress is now well defined $[5,6]$. Acute ischemia leads to increased production of free radicals and reactive oxygen species (ROS) in tissue and plasma through several mechanisms
[7], including stimulation of N-methyl-D-aspartate (NMDA) receptors [3], mitochondrial dysfunction [8], activation of neuronal nitric oxide synthase (NOS) [9], and migration of neutrophils and leukocytes that can generate superoxide anions [10]. Although its exact mechanism is not clear yet, oxidative stress is a pivotal event in the setting of ischemic stroke and may contribute to stroke outcome [11, 12].

Oxidative stress has been defined as "an imbalance between oxidants and antioxidants in favor of oxidants, 
potentially leading to damage" [13]. To assess oxidants, the accumulation of malondialdehyde (MDA), an end-product of peroxidative decomposition of polyenic fatty acids in the lipid peroxidation process, in tissues is indicative of the extent of lipid peroxidation. Measured as thiobarbituric acidreactive substances (TBARS), MDA is used as an indicator of oxidative damage for several diseases [14].

On the other hand, the antioxidant defense system has been studied in stroke patients as regards enzymes, including superoxide dismutase (SOD) and glutathione peroxidase [15, 16], and nonenzymatic antioxidants like retinol, ascorbic acid, $\alpha$-tocopherol, carotenoids, and uric acid [3, 17, 18]. Most of these studies are cross-sectional or have short follow-up periods after stroke.

Under the hypothesis that the level of oxidative stress is increased and may be diverse in different subtypes of stroke, this study evaluated longitudinal changes in serum oxidant and antioxidant levels after ischemic stroke to determine their value in predicting short-term outcome. Serial changes of serum TBARS and free thiol were measured in different subtypes during the first month after stroke and the possibility of using these markers for predicting three-month outcome was assessed.

\section{Patients and Methods}

2.1. Subjects and Design. From August 2010 to July 2012, consecutive patients with AIS who were admitted to the Neurology Department of Chang Gung Memorial HospitalKaohsiung were evaluated. Acute ischemic stroke was defined as an acute onset loss of focal cerebral function persisting for at least 24 hours. Diagnosis was based on clinical presentation, neurologic examination, and results of brain magnetic resonance imaging (MRI) with diffusion-weighted imaging (DWI). Patients aged $18-80$ years with acute noncardioembolic ischemic stroke were included and divided into two major etiologic subtypes (i.e., large-artery atherosclerosis and small-artery occlusion) according to the TOAST (Trial of Org 10172 in Acute Stroke Treatment) classification [19]. For comparison, 80 age- and sex-matched subjects with no clinical evidence of acute cerebral infarction within one year were enrolled as the control group. The hospital's Institutional Review Committee on Human Research approved the study protocol and all participants provided informed consent.

Patients with cardioembolic stroke, other determined causes and undetermined causes of stroke, and those with underlying neoplasm, end-stage renal disease, liver cirrhosis, and congestive heart failure were excluded. Clinical examination, electrocardiography, and cardiac ultrasound were conducted to exclude cardioembolic stroke. Patients with fever or any infectious disorder within the first week after acute stroke were also excluded.

2.2. Clinical Assessments and Treatment. All of the participants underwent complete neurologic examination. Brain MRI with DWI, extracranial carotid sonography, and transcranial color-coded sonography were performed during the hospitalization. The therapeutic regimens for AIS were based on the American Heart Association (AHA)/American Stroke Association (ASA) guidelines [20]. Neurologic deficits due to stroke were assessed using the National Institutes of Health Stroke Scale (NIHSS). The therapeutic outcomes were evaluated by the modified Rankin Scale (mRS) at three months after stroke. Good outcome was defined as a threemonth mRS of 0-2 without any cardiovascular event. Poor outcome was defined as $\mathrm{mRS}$ of 3-6 [21].

\subsection{Determination of Serum Malondialdehyde Content.} Blood samples were collected by venipuncture of forearm veins from patients within 48 hours of the stroke (presented as day 1) and on days 7 and 30 after stroke. Serum MDA was measured using the TBARS assay. The concentration of TBARS was assessed based on the method of Huang et al. [22]. TBARS reagent $(1 \mathrm{~mL})$ was added to a $0.5 \mathrm{~mL}$ aliquot of serum and heated for 20 minutes at $100^{\circ} \mathrm{C}$. The antioxidant, butylated hydroxytoluene, was added before heating the samples. After cooling on ice, the samples were centrifuged at $840 \mathrm{~g}$ for $15 \mathrm{~min}$. Absorbance of the supernatant was read at $532 \mathrm{~nm}$. Blanks for each sample were prepared and assessed in the same way to correct for the contribution of A532 to the sample. The TBARS results were expressed as MDA equivalents using 1,1,3,3-tetraethoxypropane.

2.4. Assessment of Serum Free Thiol Content. The ability of antioxidative defense in response to increased oxidative damage was evaluated by measuring the serum level of total reduced thiols because thiols were physiologic free radical scavengers. Serum free thiols were determined by directly reacting thiols with 5,5-dithiobis 2-nitrobenzoic acid (DTNB) to form 5-thio-2-nitrobenzoic acid (TNB). The amount of thiols in the sample was calculated from absorbance, as determined using the extinction coefficient of $\operatorname{TNB}\left(\mathrm{A} 412=13,600 \mathrm{M}^{-1} \mathrm{~cm}^{-1}\right)$.

2.5. Statistical Analysis. Data were presented as mean \pm SEM. Continuous variables, including age, cell count, lipid profile, hemoglobin Alc (HbAlc), blood pressure, and serum free thiol and TBARS, were analyzed by independent $t$-test among groups. Chi-square test or Fisher's exact test was used to compare proportions among groups. Repeated measures of ANOVA were used to compare serum free thiol and TBARS at different time points (within 48 hours and on days 7 and 30 after stroke).

Scheffe's multiple comparison was used to analyze the intraindividual courses of parameters over time. These were then compared among patients with small-vessel and largevessel diseases. Multiple logistic regression analyses determined the independent influence of different predictive variables on clinical outcome. Statistical significance was set at $P<0.05$. All statistical calculations were performed using the SAS software package, version 9.1 (2002, SAS Statistical Institute, Cary, NC, USA). 
TABLE 1: Baseline characteristics and laboratory data between small-vessel and large-vessel disease.

\begin{tabular}{lccc}
\hline & $\begin{array}{c}\text { Small vessel disease } \\
(n=75)\end{array}$ & $\begin{array}{c}\text { Large vessel disease } \\
(n=25)\end{array}$ & $\begin{array}{c}P \text { value } \\
\text { Age }(\mathrm{y})(\text { mean } \pm \mathrm{SD})\end{array}$ \\
Sex $($ male $)(n)$ & $61.1 \pm 11.5$ & $64.6 \pm 8.8$ & 0.17 \\
Hypertension $(n)$ & 60 & 22 & 0.27 \\
Diabetes mellitus $(n)$ & 56 & 10 & 0.26 \\
Dyslipidemia $(n)$ & 32 & 12 & 0.82 \\
Coronary artery disease $(n)$ & 37 & $8.1 \pm 0.5$ \\
White blood cells $\left(\times 10^{3} / \mathrm{mL}\right)$ & 5 & $4.5 \pm 0.1$ \\
Red blood cells $\left(\times 10^{6} / \mathrm{mL}\right)$ & $7.4 \pm 0.3$ & $19.8 \pm 1.2$ \\
Platelet counts $\left(\times 10^{4} / \mathrm{mL}\right)$ & $4.8 \pm 0.1$ & $194.6 \pm 11.6$ & 0.91 \\
Total cholesterol $(\mathrm{mg} / \mathrm{dL})$ & $21.1 \pm 0.7$ & $123.4 \pm 9.9$ \\
LDL-cholesterol $(\mathrm{mg} / \mathrm{dL})$ & $181.2 \pm 3.8$ & $134.9 \pm 13.3$ \\
Triglyceride $(\mathrm{mg} / \mathrm{dL})$ & $109.9 \pm 3.7$ & $6.4 \pm 0.3$ \\
HbAlc $(\%)$ & $134.7 \pm 7.7$ & $0.81 \pm 0.06$ \\
Free thiol $(\mu \mathrm{M} / \mathrm{L})$ & $7.0 \pm 0.2$ & $20.7 \pm 2.6$ \\
TBARS $(\mu \mathrm{M} / \mathrm{L})$ & $0.90 \pm 0.03$ & 0.22 \\
\hline
\end{tabular}

\section{Results}

3.1. Baseline Characteristics of Patients with Small-Vessel and Large-Vessel Diseases. Of the 120 patients with acute noncardioembolic ischemic stroke, 20 were excluded for various infections or fever in the first week after acute stroke $(n=$ $6)$, cardioembolic stroke $(n=5)$, end-stage renal disease $(n=5)$, and gastrointestinal bleeding in the acute stage $(n=$ 4). The remaining 100 patients included 75 with small-vessel occlusion and 25 with large-vessel atherosclerosis. Based on their baseline characteristics and laboratory data (Table 1), there were no significant differences in vascular risk factors and in white blood cell (WBC), red blood cell (RBC) count, platelet count, and serum levels of total cholesterol, LDLcholesterol, triglyceride, and HbAlc. Serum concentration of free thiol and TBARS were also not different between the two groups.

\subsection{Changes in Serum TBARS and Free Thiol among Patients} with Small-Vessel and Large-Vessel Diseases. Serial changes in serum concentration of TBARS among patients groups and in the controls (Figure 1) revealed that the concentration of TBARS was significantly higher in stroke patients than in the controls on days 1 and 7 after AIS $(P<0.05)$. At the three different time points, the levels of TBARS were similar between patients with large-vessel disease and those with small-vessel disease.

Serial changes in serum concentration of free thiol among patients with small-vessel and large-vessel diseases and in the controls (Figure 2) demonstrated that the concentration of free thiol was significantly lower in stroke patients than in the controls on day 1 after AIS $(P<0.05)$. Free thiol concentration was also significantly lower in the large-vessel disease group than in the small-vessel disease group on day 7 after stroke $(P<0.05)$. Thereafter, the level of free thiol gradually increased until it became similar to that of

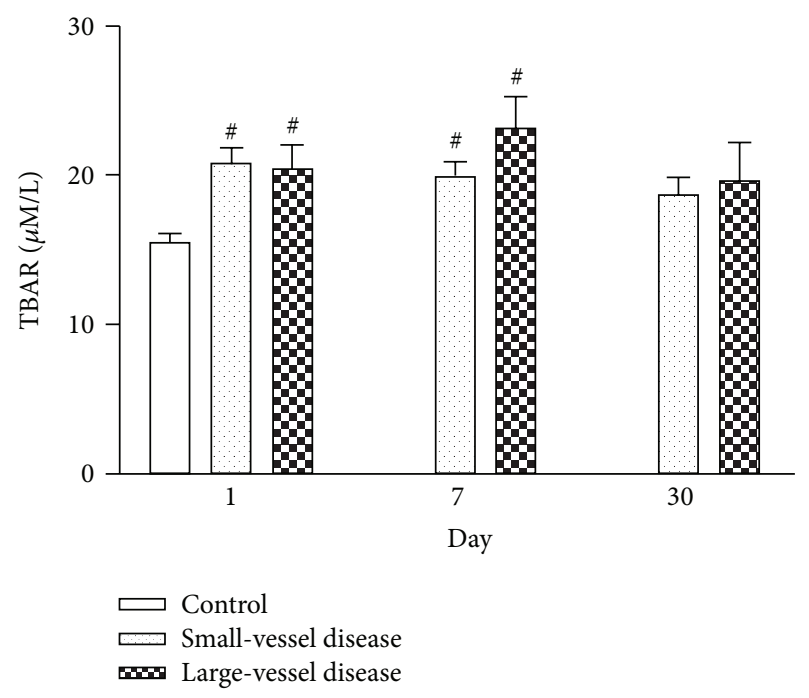

FIGURE 1: Serial changes in serum TBARS among patients with small-vessel and large-vessel diseases and in the controls at various time points after stroke. ${ }^{\#} P<0.05$ compared to controls.

the controls on day 30 after stroke. Repeated ANOVA with Scheffe's multiple comparison showed significantly different free thiol levels between patients with small-vessel disease and those with large-vessel disease at three different time points (within $48 \mathrm{~h}$ and on days 7 and 30 after stroke) $(P<$ 0.05).

3.3. Factors Predictive of Clinical Outcome. Potential prognostic factors of the 100 stroke patients were listed in Table 2. No one died during the three-month followup, and 80 patients had good outcomes while 20 had poor outcomes. Statistical analysis revealed that stroke subtype, NIHSS score, and serum free thiol and TBARS levels on days 1 and 7 after 
TABLE 2: Prognostic factors in patients with acute ischemic stroke.

\begin{tabular}{|c|c|c|c|c|c|c|}
\hline & $\begin{array}{l}\text { Good outcome } \\
\quad(n=80)\end{array}$ & $\begin{array}{l}\text { Poor outcome } \\
\quad(n=20)\end{array}$ & $\begin{array}{c}\text { Crude OR } \\
(95 \% \text { CI })\end{array}$ & $P$ value & $\begin{array}{c}\text { Adjusted OR } \\
(95 \% \mathrm{CI})\end{array}$ & $P$ value \\
\hline Age (year) & $61.2 \pm 11.5$ & $65.1 \pm 8.0$ & $1.04(0.99-1.09)$ & 0.16 & - & - \\
\hline Sex (male) $(n)$ & 62 & 15 & $0.87(0.28-2.72)$ & 0.81 & - & - \\
\hline Hypertension $(n)$ & 61 & 17 & $1.77(0.47-6.68)$ & 0.55 & - & - \\
\hline Diabetes mellitus $(n)$ & 32 & 10 & $1.50(0.56-4.01)$ & 0.46 & - & - \\
\hline Hyperlipidemia $(n)$ & 38 & 11 & $1.35(0.51-3.61)$ & 0.62 & - & - \\
\hline Cardiac disease $(n)$ & 5 & 2 & $1.67(0.30-9.30)$ & 0.80 & - & - \\
\hline NIHSS score on admission & & & $1.35(1.16-1.58)$ & $<0.001$ & $1.55(1.11-2.16)$ & 0.01 \\
\hline Stroke subtype (large/small) & & & $21.0(6.26-70.4)$ & $<0.001$ & $0.01(0.001-0.33)$ & 0.008 \\
\hline Small vessel disease & 70 & 5 & & & - & - \\
\hline Large vessel disease & 10 & 15 & & & - & - \\
\hline With statin therapy & 35 & 11 & $1.49(0.56-4.00)$ & 0.46 & - & - \\
\hline Laboratory data on admission & & & & & - & - \\
\hline White blood cells $\left(\times 10^{3} / \mathrm{mL}\right)$ & $7.4 \pm 0.2$ & $8.3 \pm 0.6$ & $1.17(0.96-1.43)$ & 0.12 & - & - \\
\hline Hemoglobin $(\mathrm{g} / \mathrm{dL})$ & $13.8 \pm 0.2$ & $13.6 \pm 0.3$ & $0.91(0.69-1.22)$ & 0.53 & & \\
\hline Red blood cells $\left(\times 10^{6} / \mathrm{mL}\right)$ & $4.8 \pm 0.1$ & $4.5 \pm 0.1$ & $0.50(0.24-1.07)$ & 0.07 & - & - \\
\hline Platelet counts $\left(\times 10^{4} / \mathrm{mL}\right)$ & $21.2 \pm 0.6$ & $18.9 \pm 1.4$ & $0.99(0.98-1.00)$ & 0.12 & - & - \\
\hline Total cholesterol (mg/dL) & $185.7 \pm 4.6$ & $180.0 \pm 8.6$ & $0.99(0.98-1.01)$ & 0.58 & - & - \\
\hline LDL-cholesterol (mg/dL) & $114.2 \pm 4.2$ & $109.6 \pm 7.7$ & $0.99(0.98-1.01)$ & 0.62 & - & - \\
\hline HDL-cholesterol (mg/dL) & $43.8 \pm 1.0$ & $45.6 \pm 3.0$ & $1.02(0.97-1.07)$ & 0.48 & - & - \\
\hline Triglyceride (mg/dL) & $136.3 \pm 7.6$ & $128.3 \pm 13.8$ & $0.99(0.99-1.01)$ & 0.63 & - & - \\
\hline $\operatorname{HbAlc}(\%)$ & $6.8 \pm 0.2$ & $7.3 \pm 0.6$ & $1.14(0.90-1.45)$ & 0.28 & - & - \\
\hline Systolic BP (mmHg) & $147.1 \pm 2.8$ & $140.1 \pm 4.4$ & $0.99(0.97-1.01)$ & 0.28 & - & - \\
\hline Diastolic BP (mmHg) & $84.5 \pm 1.5$ & $81.1 \pm 2.8$ & $0.98(0.94-1.02)$ & 0.31 & - & - \\
\hline Free thiol on admission $(\mu \mathrm{M} / \mathrm{L})$ & $0.92 \pm 0.03$ & $0.70 \pm 0.07$ & $0.04(0.01-0.32)$ & 0.002 & - & - \\
\hline TBARS on admission $(\mu \mathrm{M} / \mathrm{L})$ & $19.9 \pm 0.72$ & $24.1 \pm 3.0$ & $1.05(0.99-1.11)$ & 0.06 & - & - \\
\hline Free thiol on day $7(\mu \mathrm{M} / \mathrm{L})$ & $0.96 \pm 0.04$ & $0.66 \pm 0.08$ & $0.12(0.03-0.52)$ & 0.005 & - & - \\
\hline TBARS on day $7(\mu \mathrm{M} / \mathrm{L})$ & $18.4 \pm 0.77$ & $29.1 \pm 2.8$ & $1.14(1.07-1.22)$ & $<0.001$ & $1.37(1.14-1.65)$ & 0.001 \\
\hline Free thiol on day $30(\mu \mathrm{M} / \mathrm{L})$ & $1.00 \pm 0.04$ & $0.88 \pm 0.11$ & $0.29(0.05-1.75)$ & 0.78 & - & - \\
\hline TBARS on day $30(\mu \mathrm{M} / \mathrm{L})$ & $19.7 \pm 1.24$ & $20.4 \pm 2.4$ & $1.01(0.95-1.07)$ & 0.82 & - & - \\
\hline
\end{tabular}

Abbreviations: BP: blood pressure; HbAlc: hemoglobin Alc.

stroke were significantly different between the good and poor outcome groups.

Potential variables such as age, sex, stroke subtype, blood pressure, HbA1C, total cholesterol, HDL, LDL, and TBARS and free thiol levels on admission were analyzed using a stepwise logistic regression model. Only the stroke subtype (OR: 0.014 , 95\% CI: 0.001-0.325; $P=0.008$ ), NIHSS score (OR: 1.55 , 95\% CI: 1.11-2.16; $P=0.01$ ), and serum TBARS on day 7 after stroke (OR: 1.37, 95\% CI: 1.14-1.65; $P=0.001$ ) were independently associated with three-month outcome. Any increase in TBARS concentration by one $\mu \mathrm{M} / \mathrm{L}$ worsens the poor outcome rate by $37 \%$.

3.4. Serial Changes in Serum TBARS and Free Thiol between Groups with Good and Poor Outcomes. Changes in serum TBARS between groups with good and poor outcomes (Figure 3) revealed a gross increase in TBARS level in the poor outcome group during the acute stage after stroke. The concentration of TBARS was significantly higher in the poor outcome group than in the good outcome group on day 7 after stroke $(P<0.05)$. These levels gradually decreased thereafter and no significant difference existed between the two groups on day 30 after stroke. Repeated ANOVA with Scheffe's multiple comparison revealed significantly different serum TBARS levels on three different time points between the two groups $(P<0.05)$.

Changes in serum concentration of free thiol among patients with good and poor outcomes, and in the controls (Figure 4) revealed that the concentration of free thiol was significantly lower in the poor outcome group than in the good outcome group on days 1 and 7 after stroke $(P<$ 0.05). Repeated ANOVA with Scheffe's multiple comparison showed significantly different free thiol levels between the good and poor outcome groups at three different time points (within $48 \mathrm{~h}$ and on days 7 and 30 after stroke) $(P<0.05)$. 

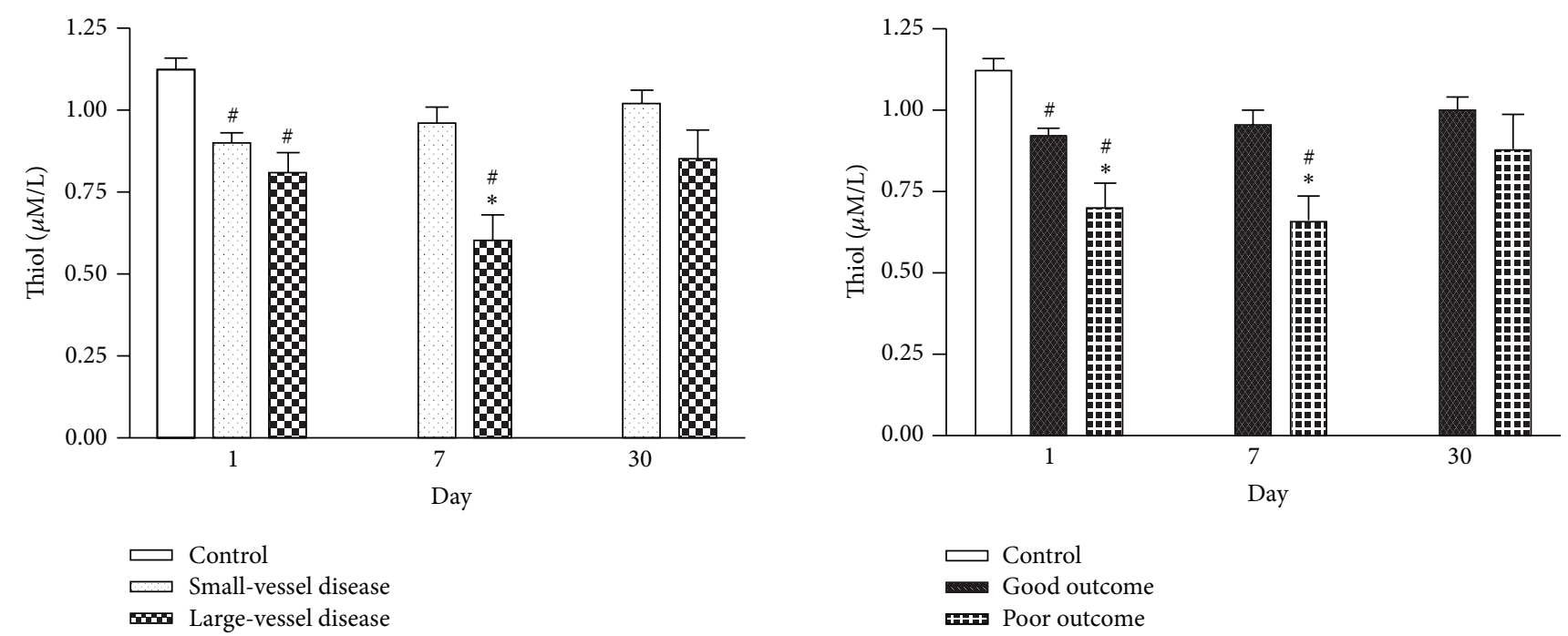

FIGURE 2: Serial changes in serum free thiol among patients with small-vessel and large-vessel diseases and in the controls at various time points after stroke. ${ }^{*} P<0.05$ compared to the small vessel group; ${ }^{\#} P<0.05$ compared to the controls.

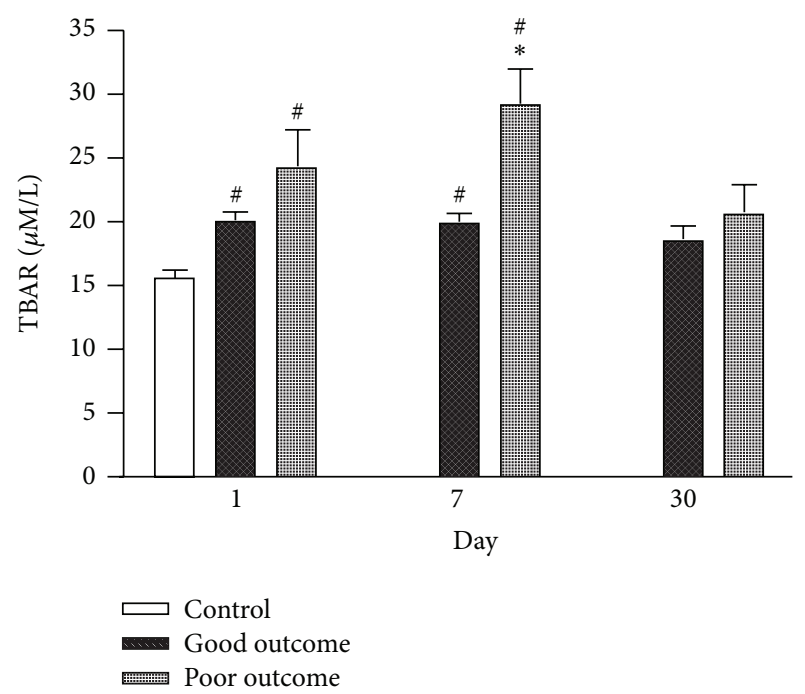

FIGURE 3: Serial changes in serum TBARS among patients with good and poor outcomes and in the controls at various time points after stroke. ${ }^{*} P<0.05$ compared to the small vessel group; ${ }^{\#} P<0.05$ compared to the controls.

\section{Discussion}

The present study has four major findings. First, patients with AIS in the acute phase had significantly higher TBARS and lower free thiol levels than the controls. Second, the level of free thiol is significantly lower in patients with large-vessel disease than in those with small-vessel disease on day 7 after stroke. Third, the higher TBARS and lower free thiol levels in the acute phase of AIS is associated with poor outcome. Lastly, the most important finding in this study is that TBARS level on day 7 after stroke is an independent predictive factor of three-month outcome.
FIgURE 4: Serial changes in serum free thiol among patients with good and poor outcomes and in the controls at various time points after stroke. ${ }^{*} P<0.05$ compared to the small vessel group; ${ }^{\#} P<$ 0.05 compared to the controls.

The results on the MDA level changes over time in stroke patients are controversial. Some researchers observed higher erythrocyte MDA levels in the very early phase of stroke, with subsequent decline in the levels of the controls [23], while others report an MDA increase only in some days after acute stroke [24]. Gariballa et al. found that TBARs levels were constantly higher in AIS patients compared to those of the controls [15]. Consistent with a previous study [25], the longitudinal observation here shows higher levels of TBARS in stroke patients than in controls upon hospital admission, and these levels persist in the next 7 days. Thereafter, TBARS levels gradually decrease to the level as controls a month after the stroke. The discrepancy in results may be due to methodological factors, including sample handling, storage, and preparation prior to the performance of the biomarker assay. Moreover, stroke is a pathologically heterogeneous disease and baseline risk factors may be etiologically different.

Furthermore, the current study demonstrates that antioxidant levels, measured by free thiol, are much lower in patients with large-vessel cerebral infarction than in those with small-vessel infarction on day 7 after stroke. The level of free thiol gradually increases until the difference is no longer significant one month after the stroke. These suggest that oxidant/antioxidant balance is related to the different pathogenesis in the two major subtypes of noncardioembolic stroke. The pathogenesis of small-vessel infarction is lipohyalinosis [26], while atherothrombosis is the major cause of large-vessel cerebral infarction [27]. Thus, different subtypes of ischemic stroke have different pathogenesis, with consequences on oxidative stress.

Oxidative stress is an important contributor to the pathophysiologic sequelae of stroke. A correlation of MDA level with infarct size, clinical stroke severity, and patient outcome has been observed $[15,25]$. Since plasma level of oxidized LDL (Ox-LDL) is thought to reflect the oxidative status of the whole body, a previous study has shown that higher Ox-LDL 
level in the acute phase of AIS is an independent predictor of poor outcome three months after stroke [28]. The results here reveal that the level of TBARS on day 7 but not day 1 after stroke is much higher in patients with poor outcome than in those with good outcome (Figure 3). Moreover, TBARS level on day 7 after stroke is an independent predictive factor of three-month outcome. These findings suggest that oxidative stress is progressive after stroke and contributes to further neurologic damage in particular cases.

On the other hand, antioxidant vitamin concentrations are associated with neurologic damage and stroke prognosis $[29,30]$. The antioxidant defense system, measured by SOD activity, is inversely correlated with infarct size and the severity of neurologic damage $[15,16]$. The data here also confirms that the lower antioxidant (free thiol) level in the acute phase of stroke is associated with poor outcome.

Although other inflammatory biomarkers like highsensitivity C-reactive protein (hs-CRP) and leukocytes have been reported to be useful in predicting clinical outcome after stroke [31, 32], there has been no head-to-head study to date that compares these markers in terms of predictive value. A previous research demonstrates that statin therapy reduces serum hs-CRP level and oxidized LDL in patients after AIS [33]. A prospective study is warranted to evaluate the predictive value of these biomarkers on stroke outcome.

Some limitations of this study should be acknowledged. First, the measurement of only few biomarkers of oxidative damage cannot be considered a valid tool for exploring a multifaceted, complex oxidant/antioxidant imbalance after acute stroke. Second, the oxidant/antioxidant balance of stroke patients may be influenced by a multitude of parameters, including age, sex, smoking habit, alcohol consumption, physical activity, and vitamin supplementation. Third, oxidative stress may be influenced by other drugs (e.g., antiplatelet, angiotensin II type 1 receptor blockers, and antidiabetics). Since the use of these drugs depends on the preference of the attending physician, this may cause potential bias in statistical analysis and in drawing conclusions. Nonetheless, the sample size is not large and the number of variables considered in the stepwise logistic regression analysis is small. Hence, the maximum likelihood estimates of the coefficients are valid in the analysis.

In conclusion, patients with AIS have significantly higher TBARS and lower free thiol levels than healthy controls. The level of free thiol is significantly lower in patients with largevessel disease than in those with small-vessel disease in the acute phase of stroke. Serum TBARS on day 7 after stroke is an independent predictive biomarker of three-month stroke outcome.

\section{Conflict of Interests}

All of the authors declared that they have no conflict of interests.

\section{Acknowledgments}

This study was supported by grants from the National Science Council Research Project (99-2314-B-182-055-MY2).
The authors wish to thank Doctor Gene Alzona Nisperos who provided language help and writing assistance for this paper.

\section{References}

[1] N. Suwanwela and W. J. Koroshetz, "Acute ischemic stroke: overview of recent therapeutic developments," Annual Review of Medicine, vol. 58, pp. 89-106, 2007.

[2] Y. Samson, B. Lapergue, and H. Hosseini, "Inflammation and ischaemic stroke: current status and future perspectives," Revue Neurologique, vol. 161, no. 12, pp. 1177-1182, 2005.

[3] A. Cherubini, C. Ruggiero, M. C. Polidori, and P. Mecocci, "Potential markers of oxidative stress in stroke," Free Radical Biology and Medicine, vol. 39, no. 7, pp. 841-852, 2005.

[4] T. Goodman, P. Sharma, and A. Ferro, "The genetics of aspirin resistance," International Journal of Clinical Practice, vol. 61, no. 5, pp. 826-834, 2007.

[5] L. C. Álvaro-González, M. M. Freijo-Guerrero, and F. SádabaGaray, "Inflammatory mechanisms, arteriosclerosis and ischemic stroke: clinical data and perspectives," Revista de Neurologia, vol. 35, no. 5, pp. 452-462, 2002.

[6] A. Chamorro, "Role of inflammation in stroke and atherothrombosis," Cerebrovascular Diseases, vol. 17, no. 3, supplement, pp. 1-5, 2004.

[7] T. J. Guzik, R. Korbut, and T. Adamek-Guzik, "Nitric oxide and superoxide in inflammation and immune regulation," Journal of Physiology and Pharmacology, vol. 54, no. 4, pp. 469-487, 2003.

[8] M. Lafon-Cazal, S. Pietri, M. Culcasi, and J. Bockaert, "NMDAdependent superoxide production and neurotoxicity," Nature, vol. 364, no. 6437, pp. 535-537, 1993.

[9] C. A. Piantadosi and J. Zhang, "Mitochondrial generation of reactive oxygen species after brain ischemia in the rat," Stroke, vol. 27, no. 2, pp. 327-332, 1996.

[10] S. A. Lipton and P. A. Rosenberg, "Mechanisms of disease: excitatory amino acids as a final common pathway for neurologic disorders," New England Journal of Medicine, vol. 330, no. 9, pp. 613-622, 1994.

[11] C. E. Walder, S. P. Green, W. C. Darbonne et al., "Ischemic stroke injury is reduced in mice lacking a functional NADPH oxidase," Stroke, vol. 28, no. 11, pp. 2252-2258, 1997.

[12] Y. Matsuo, T. Kihara, M. Ikeda, M. Ninomiya, H. Onodera, and K. Kogure, "Role of neutrophils in radical production during ischemia and reperfusion of the rat brain: effect of neutrophil depletion on extracellular ascorbyl radical formation," Journal of Cerebral Blood Flow and Metabolism, vol. 15, no. 6, pp. 941$947,1995$.

[13] H. Sies, "Oxidative stress: oxidants and antioxidants," Experimental Physiology, vol. 82, no. 2, pp. 291-295, 1997.

[14] F. Karatas, M. Karatepe, and A. Baysar, "Determination of free malondialdehyde in human serum by high-performance liquid chroma tography," Analytical Biochemistry, vol. 311, no. 1, pp. 7679, 2002.

[15] S. E. Gariballa, T. P. Hutchin, and A. J. Sinclair, "Antioxidant capacity after acute ischaemic stroke," QJM, vol. 95, no. 10, pp. 685-690, 2002.

[16] M. Spranger, S. Krempien, S. Schwab, S. Donneberg, and W. Hacke, "Superoxide dismutase activity in serum of patients with acute cerebral ischemic injury: correlation with clinical course and infarct size," Stroke, vol. 28, no. 12, pp. 2425-2428, 1997.

[17] C.-Y. Chang, Y.-C. Lai, T.-J. Cheng, M.-T. Lau, and M.-L. $\mathrm{Hu}$, "Plasma levels of antioxidant vitamins, selenium, total 
sulfhydryl groups and oxidative products in ischemic-stroke patients as compared to matched controls in Taiwan," Free Radical Research, vol. 28, no. 1, pp. 15-24, 1998.

[18] J. M. Leppälä, J. Virtamo, R. Fogelholm, D. Albanes, and O. P. Heinonen, "Different risk factors for different stroke subtypes: association of blood pressure, cholesterol, and antioxidants," Stroke, vol. 30, no. 12, pp. 2535-2540, 1999.

[19] H. P. Adams Jr., B. H. Bendixen, L. J. Kappelle et al., "Classification of subtype of acute ischemic stroke: definitions for use in a multicenter clinical trial," Stroke, vol. 24, no. 1, pp. 35-41, 1993.

[20] R. L. Sacco, R. Adams, G. Albers et al., "Guidelines for prevention of stroke in patients with ischemic stroke or transient ischemic attack: a statement for healthcare professionals from the American Heart Association/American Stroke Association council on stroke-co-sponsored by the council on cardiovascular radiology and intervention. The American Academy of Neurology affirms the value of this guideline," Stroke, vol. 37, no. 2, pp. 577-617, 2006.

[21] G. Sulter, C. Steen, and J. De Keyser, "Use of the Barthel Index and Modified Rankin Scale in acute stroke trials," Stroke, vol. 30, no. 8, pp. 1538-1541, 1999.

[22] T.-L. Huang, C.-W. Liou, and T.-K. Lin, "Serum thiobarbituric acid-reactive substances and free thiol levels in schizophrenia patients: effects of antipsychotic drugs," Psychiatry Research, vol. 177, no. 1-2, pp. 18-21, 2010.

[23] S. Demirkaya, M. A. Topcuoglu, A. Aydin, U. H. Ulas, A. I. Isimer, and O. Vural, "Malondialdehyde, glutathione peroxidase and superoxide dismutase in peripheral blood erythrocytes of patients with acute cerebral ischemia," European Journal of Neurology, vol. 8, no. 1, pp. 43-51, 2001.

[24] P. C. Sharpe, C. Mulholland, and T. Trinick, "Ascorbate and malondialdehyde in stroke patients," Irish Journal of Medical Science, vol. 163, no. 11, pp. 488-491, 1994.

[25] M. C. Polidori, A. Cherubini, W. Stahl, U. Senin, H. Sies, and P. Mecocci, "Plasma carotenoid and malondialdehyde levels in ischemic stroke patients: relationship to early outcome," Free Radical Research, vol. 36, no. 3, pp. 265-268, 2002.

[26] G. A. Lammie, F. Brannan, J. Slattery, and C. Warlow, "Nonhypertensive cerebral small-vessel disease: an autopsy study," Stroke, vol. 28, no. 11, pp. 2222-2229, 1997.

[27] P. M. Rothwell, "Atherothrombosis and ischaemic stroke," British Medical Journal, vol. 334, no. 7590, pp. 379-380, 2007.

[28] N. W. Tsai, L. H. Lee, C. R. Huang et al., "Statin therapy reduces oxidized low density lipoprotein level, a risk factor for stroke outcome," Critical Care, vol. 18, no. 1, article R16, 2014.

[29] J. de Keyser, N. de Klippel, H. Merkx, M. Vervaeck, and L. Herroelen, "Serum concentrations of vitamins A and E and early outcome after ischaemic stroke," The Lancet, vol. 339, no. 8809, pp. 1562-1565, 1992.

[30] J. S. Leinonen, J.-P. Ahonen, K. Lönnrot et al., "Low plasma antioxidant activity is associated with high lesion volume and neurological impairment in stroke," Stroke, vol. 31, no. 1, pp. 3339, 2000.

[31] T. T. Idicula, J. Brogger, H. Naess, U. Waje-Andreassen, and L. Thomassen, "Admission C-reactive protein after acute ischemic stroke is associated with stroke severity and mortality: the 'Bergen stroke study,' BMC Neurology, vol. 9, article 18, 2009.

[32] M. S. V. Elkind, J. Cheng, T. Rundek, B. Boden-Albala, and R. L. Sacco, "Leukocyte count predicts outcome after ischemic stroke: the Northern Manhattan Stroke Study," Journal of Stroke and Cerebrovascular Diseases, vol. 13, no. 5, pp. 220-227, 2004.
[33] N. W. Tsai, L. H. Lee, and C. R. Huang, "The association of statin therapy and high-sensitivity C-reactive protein level for predicting clinical outcome in acute non-cardio-embolic ischemic stroke," Clinica Chimica Acta, vol. 413, no. 23-24, pp. 1861-1865, 2012. 

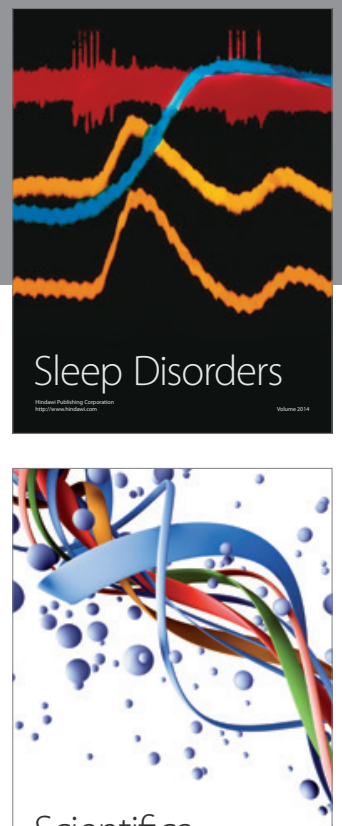

Scientifica
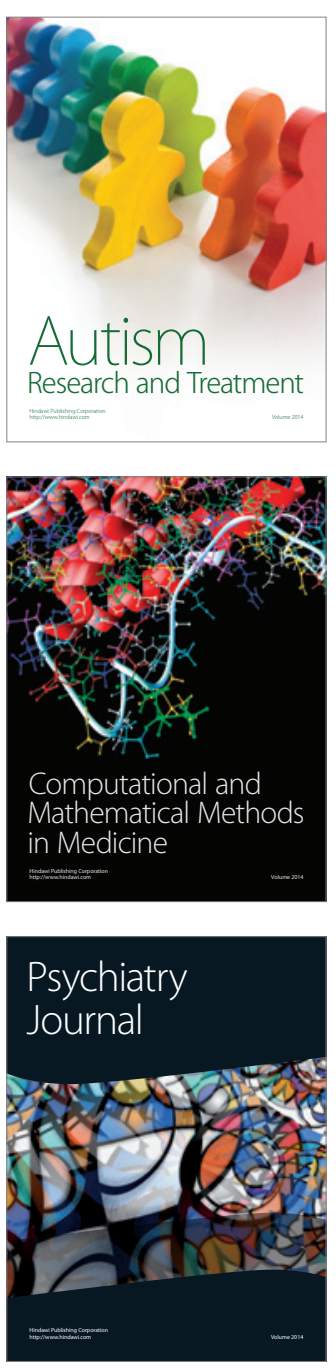
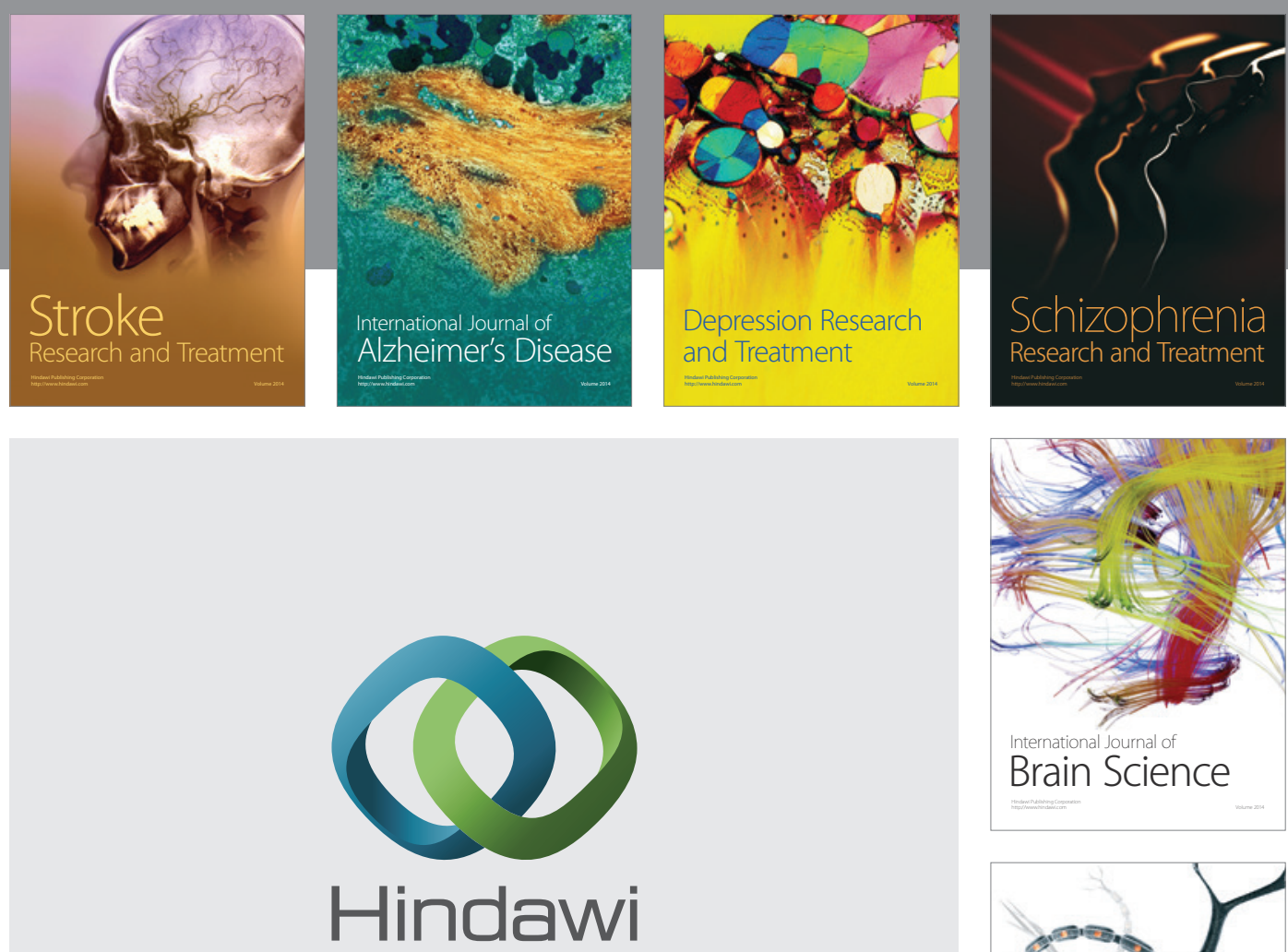

Submit your manuscripts at

http://www.hindawi.com
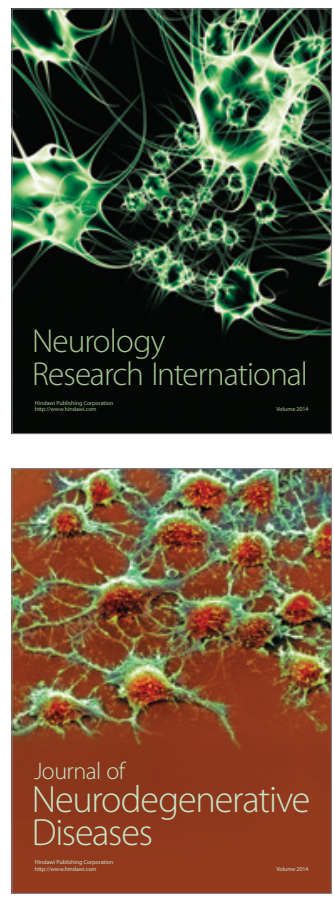

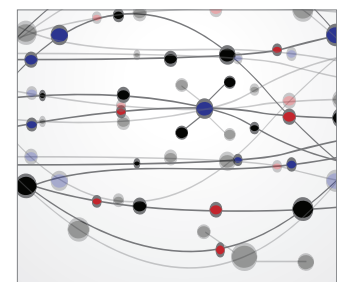

The Scientific World Journal
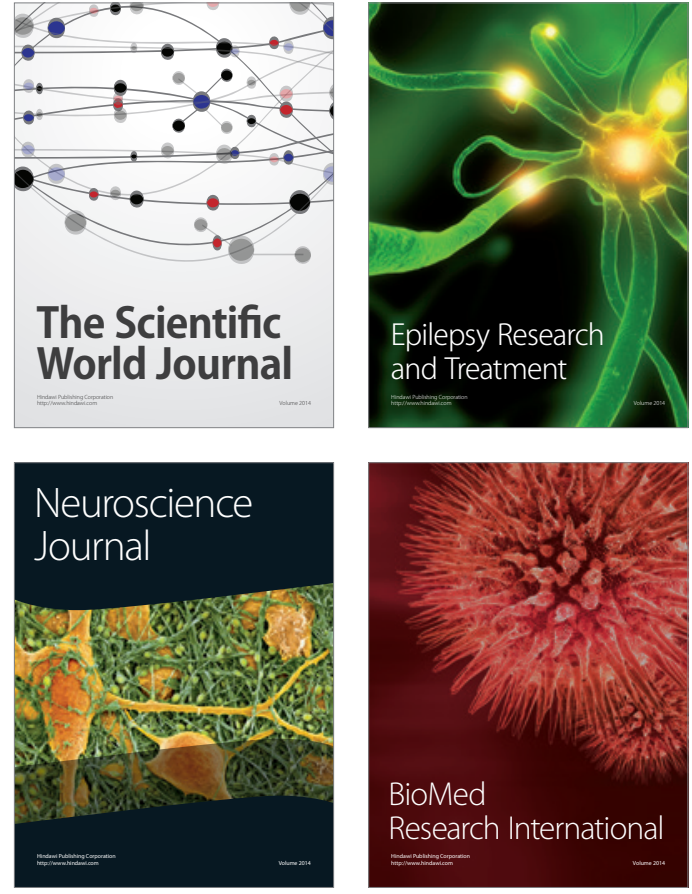

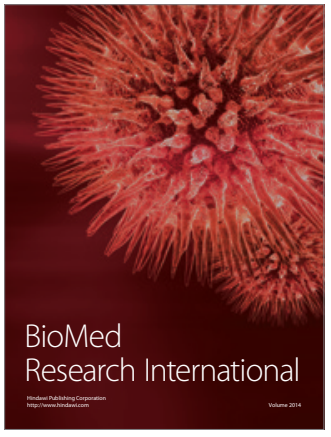

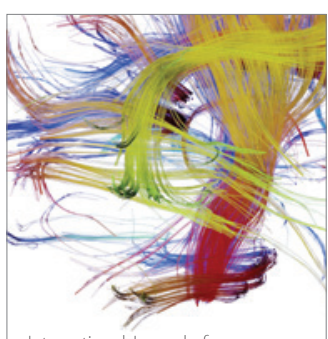

Brain Science

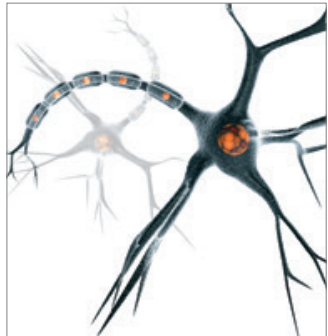

Neural Plasticity
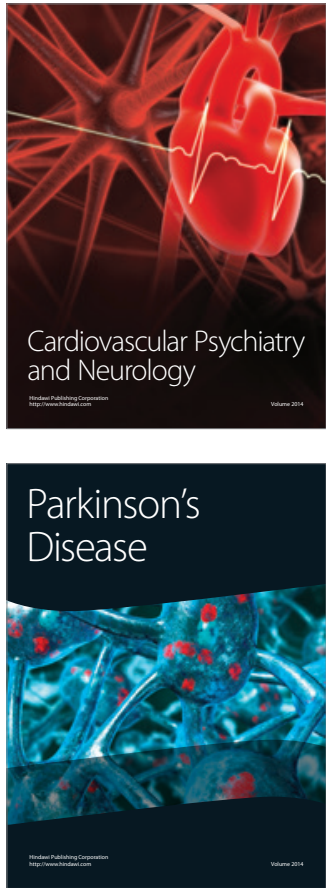